\title{
Should Cushing Syndrome Be Only Evaluated by Endocrinologists and Neurosurgeons?
}

\section{Çiğdem TURA BAHADIR, Feyzi GÖKOSMANOĞLU, Elif KILIÇ \\ KAN, Gülçin CENGIZ ECEMIŞ, Ayșegül ATMACA, Hulusi \\ ATMACA, Ramis ÇOLAK \\ Ondokuz Mayıs University School of Medicine \\ Department of Endocrionology and Metabolism \\ Samsun, TURKEY}

\section{OBJECTIVES}

Physical features such as central obesity, purple stria, thin skin, moon face and buffalo hump may be observed in Cushing's syndrome (CS). Psychiatric and psychological disturbances may also be present in addition to the physical problems. The most common mental disturbance is major depression. Mania and anxiety disorders may also be seen. It may be detected both in active period or in remission of CS on account of persistant effect of previous period of hypercortisolism, hypopituitarism and glucocorticoid deficiency. In this study, we aimed to analyze the frequency of psychopathologic disorders in CS.

\section{METHODS}

We prospectively followed 62 patients that had been diagnosed with CS between 2010 and 2014 in our clinic. Of the patients, 60\% were cushing's disease (CD) (29 female, 8 male) and $40 \%$ were ACTH-independent CS (20 female, 5 male). The patients who had been under medication for psychopathology were included in the study.

\begin{tabular}{|c|c|c|c|c|c|c|c|}
\hline $\begin{array}{l}\text { Etiology } \\
\text { of CS }\end{array}$ & $\begin{array}{l}\text { Age- } \\
\text { Sex }\end{array}$ & $\begin{array}{l}\text { Diagnosis time } \\
\text { of depression }\end{array}$ & $\begin{array}{c}\text { Probable reason of } \\
\text { depression }\end{array}$ & $\begin{array}{l}\text { Suicide } \\
\text { attempt }\end{array}$ & $\begin{array}{l}\text { Cortisole } \\
\text { after } \\
\text { LDDST } \\
(\mu \mathrm{g} / \mathrm{dl}) \\
\end{array}$ & $\begin{array}{l}\text { ACTH } \\
(\mathrm{pg} / \mathrm{ml})\end{array}$ & SCP \\
\hline A & $27-\mathrm{F}$ & Before surgery & active disease & - & 4,5 & 15 & $\overline{-}$ \\
\hline A & $23-\mathrm{F}$ & After surgery & adrenal insufficiency & After surgery* & 31 & $<5$ & + \\
\hline A & $55-\mathrm{F}$ & Before surgery & active disease & - & 15 & 25 & - \\
\hline$C D$ & $29-\mathrm{F}$ & Before surgery & active disease & - & 12 & 65 & - \\
\hline$C D$ & $45-\mathrm{F}$ & Before surgery & active disease & - & 16 & 65 & + \\
\hline$C D$ & $53-\mathrm{F}$ & After surgery & persistant disease & - & 18 & 82 & + \\
\hline$C D$ & $32-\mathrm{F}$ & Before surgery & active disease & - & 8 & 109 & + \\
\hline$C D$ & $35-\mathrm{F}$ & Before surgery & active disease & $\begin{array}{l}\text { After } \\
\text { surgery* }\end{array}$ & 11 & 55 & + \\
\hline$C D$ & 39-M & After surgery & $\begin{array}{l}\text { hypogonadism } \\
\text { GH deficiency } \\
\text { acromegaly } \\
\text { uncontrolled DM }\end{array}$ & - & 19 & 57 & - \\
\hline$C D$ & $44-\mathrm{F}$ & After surgery & $\begin{array}{l}\text { GH deficiency } \\
\text { decreased libido }\end{array}$ & - & 20 & 44 & + \\
\hline$C D$ & $29-\mathrm{F}$ & Before surgery & active disease & - & 15 & 66 & + \\
\hline$C D$ & $45-\mathrm{F}$ & Before surgery & active disease & - & 3 & 39 & - \\
\hline \multicolumn{8}{|c|}{$\begin{array}{l}\text { A: ACTH-independent Cushing syndrome F: Female M: Male CD: Cushing disease SCP: Severe } \\
\text { clinical presentation DM: diabetes mellitus } \\
\text { * early postoperative visual field defect and suicide attempt } 1,5 \text { year after the surgery } \\
\text { ** early postoperative visual field defect and suicide attempt } 2 \text { years after the surgery }\end{array}$} \\
\hline
\end{tabular}

\section{RESULTS}

The prevalence of psychopathology was $19 \%$ in CS $(12$ patients). In ACTH-independent CS and CD it was $12 \%$ and $24 \%$, respectively. Eleven $(92 \%)$ patients were female. Nine $(75 \%)$ patients had active diseases. Two patients, one with $\mathrm{CD}$ and one with $\mathrm{ACTH}$-independent $\mathrm{CS}$, had history of suicide attempts 1.5 and 2 years after surgery, respectively. Both suicide attempts happened after surgery. The patients who attempted suicide had glucocorticoid deficiency and hypopituitarism, respectively.

\section{CONCLUSIONS}

In CS patients, psychiatric and psychologic disorders may be seen before or after treatment. Despite treatment for CS, they still may have severe psychopathologic disorders to the degree of suicide attempts due to hormonal insufficiencies in the postoperative period that may result from medication or surgical complications. For this reason, patients should be evaluated not only by endocrinology and neurosurgery clinics, but also psychiatry clinics both before and after the surgery. 